\title{
Robust Control Design of an Electro-Hydraulic Actuator
}

\author{
Balázs Németh, Balázs Varga and Péter Gáspár
}

\begin{abstract}
The paper proposes a hierarchical control design of an electro-hydraulic actuator. The high-level hydromotor is modeled with a linear form with parametric uncertainty, while the low-level spool valve is modeled with a polynomial system. The subsystems require different control strategies. At the high level a robust $\mathcal{H}_{\infty} / \mu$ control is used in order to meet the performance specifications. At the low level a Control Lyapunov Function-based algorithm is proposed, which calculates discrete control input values for the valve. The interaction between the two control systems is guaranteed by the spool displacement, which is the control input at the high level and must be tracked at the low level. The operation of the actuator control system is illustrated through a simulation example.
\end{abstract}

\section{INTRODUCTION AND MOTIVATION}

Hydraulic actuators are used in several engineering applications, therefore, developing advanced control methods for these systems is relevant. One of these applications is active anti-roll bars, which enhance the roll stability of vehicles.

The literature of hydraulic control systems is very extensive. The robotic applications of the commonly-used electronically-controlled actuators, such as electromagnetic motors, hydraulic, pneumatic and piezoelectric actuators were detailed and compared, see e.g., [1]. A nonlinear PID controller for a hydraulic positioning system was proposed by [2]. A velocity tracking robust PID control of an hydraulic cylinder based on linear model with parameter uncertainties was published in [3]. A sliding control to deal with a highly nonlinear model was proposed by [4]. In [5] and [6] a robust low-order control design of an electro-hydraulic cylinder was presented and analyzed on a test bed. In [7] a feedback control scheme for motion control of nonlinear high-order systems was proposed. A Fuzzy control was also proposed for the design of a hydraulic cylinder, see [8].

The paper focuses on an electro-hydraulic actuator, i.e., an oscillating hydromotor and a spool valve. The oscillating hydromotor is a rotary actuator with two cells, which are separated by vanes. The pressure difference between the vanes generates a torque on the central shaft, which has a limited rotation angle. The hydromotor is connected to a symmetric $4 / 2$ four-way valve and the spool is controlled by a solenoid valve. The spool has a limited distance to travel and the input current can only take discrete values. Since the presented system has a high energy density, it requires small space and it has low mass. Besides, the actuator has a simple construction, but it requires an external high-pressure pump [9].

B. Varga, B. Németh and P. Gáspár are with Systems and Control Laboratory, Institute for Computer Science and Control, Hungarian Academy of Sciences, Kende u. 13-17, H-1111 Budapest, Hungary. E-mail: [ bnemeth; gaspar; bvarga] dsztaki.mta.hu

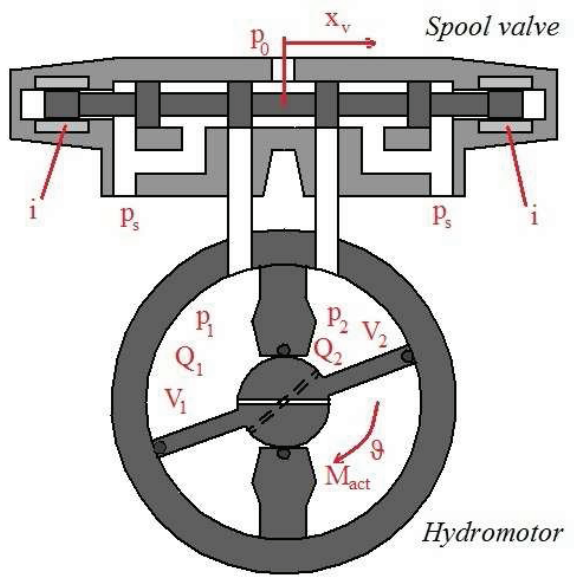

Fig. 1. Oscillating hydromotor actuator

The control-oriented model of the actuator is separated into two subsystems. The high-level hydromotor is modeled with a linear form with parametric uncertainty, while the low-level spool valve is modeled with a polynomial system. The subsystems require different control strategies. At the high level a robust $\mathcal{H}_{\infty} / \mu$ control is used in order to meet the performance specifications. At the low level a Control Lyapunov Function-based algorithm is proposed, which calculates discrete control input values for the valve. The interaction between the two control systems is guaranteed by the spool displacement, which is the control input at the high level and must be tracked at the low level.

The paper is organized as follows. Section II presents the control-oriented hydromotor and valve models. Section III proposes the control design of the spool valve. Section IV presents the hierarchical control structure and proposes the design of the robust control of the hydromotor. Section V illustrates the operation of the multi-level control system through a simulation example. Finally, Section VI gives some concluding remarks.

\section{MOdELING THE ELECTRO-HYDRAULIC ACTUATOR}

\section{A. Modeling the hydromotor}

In the following the control-oriented modeling of the hydromotor is proposed. The output of the system is the actuator torque $M_{a c t}$, which improves the roll dynamics of the vehicle. The input of the system is the electromagnetic valve motion $x_{v}$. The illustration of the hydromotor construction is found in Figure 1.

The pressures in the chambers depend on the flows of the circuits $Q_{1}, Q_{2}$. $p_{L}$ is the load pressure difference between 
the two chambers. The average flow of the system, assuming the supply pressure $p_{s}$ is constant, is as follows:

$$
Q_{L}\left(x_{v}, p_{L}\right)=C_{d} A\left(x_{v}\right) \sqrt{\frac{1}{\rho}\left(p_{s}-\frac{x_{v}}{\left|x_{v}\right|} p_{L}\right)}
$$

This equation can be linearized around $\left(x_{v, 0} ; p_{L, 0}\right)$ such as

$$
Q_{L}=K_{q} x_{v}-K_{c} p_{L}
$$

where $K_{q}$ is the valve flow gain coefficient and $K_{c}$ is the valve pressure coefficient, see [9]. In this modeling principle, the hydromotor model does not take into account the friction force and the external leakage flow. The compressibility of the fluid is function of the system pressure and the percentage of air trapped in the system. The volumetric flow in the chambers is formed as

$$
\dot{p}_{L}=\frac{4 \beta_{E}}{V_{t}}\left(Q_{L}-V_{p} \varphi+c_{l 1} \dot{\varphi}-c_{l 2} p_{L}\right)
$$

where $\beta_{E}$ is the effective bulk modulus, $V_{t}$ is the total volume under pressure and $V_{p}$ is proportional to the areas of vane cross-sections. $c_{l 1}$ and $c_{l 2}$ are parameters of the leakage flow.

The motion equation of the shaft rotation $\dot{\varphi}$ due $p_{L}$ and the external load $M_{d i s t}$ can be written as follows:

$$
J \ddot{\phi}=-d_{a} \dot{\varphi}+V_{p} p_{L}+M_{\text {dist }}
$$

where $J$ is the mass of the hydromotor shaft and vanes, $d_{a}$ is the damping constant of the system. The actuator torque $M_{a c t}$ is written as:

$$
M_{a c t}=2 A_{v} \frac{d_{e}}{2} p_{L}
$$

with $A_{v}$ being the area of the vanes and $d_{e}$ is the effective diameter of the vanes.

Using (3) and (4) the state-space representation of the hydromotor is formed as:

$$
\dot{x}_{H M}=A_{H M} x_{H M}+B_{1 H M} w+B_{2 H M} u
$$

where the state vector is $x_{H M}=\left[p_{L}, \dot{\varphi}, \varphi\right]^{T}$.

\section{B. Modeling the electromagnetic valve}

The electronically controlled spool valve is modeled in a polynomial form, which creates dependence between current $i$ and spool displacement $x_{v}$. The motion equation of the valve is written as follows:

$$
\frac{1}{\omega_{v}^{2}} \ddot{x}_{v}+\frac{2 D_{v}}{\omega_{v}} \dot{x}_{v}+x_{v}=k_{v} \omega_{v}^{2} i
$$

where $k_{v}$ valve gain equals

$$
k_{v}=\frac{Q_{N}}{\sqrt{\Delta p_{N} / 2}} \frac{1}{u_{v \max }} .
$$

$Q_{N}$ is the rated flow at rated pressure and maximum input current, $p_{N}$ is the pressure drop at rated flow and $u_{v \max }$ is the maximum rated current. $D_{v}$ is the valve damping coefficient, which can be calculated from the apparent damping ratio. $\omega_{v}$ stands for the natural frequency of the valve [8]. Let $K_{f}=\omega_{v}^{2}$, which is a spring-stiffness-like parameter. In the model the nonlinear friction of the valve is neglected.
The flow force stiffness of the system for control purposes is approximated as [9]

$$
K_{f}\left(x_{v}\right) \approx 0.43\left(p_{s}-p_{L}\right) \cdot w\left(x_{v}\right)
$$

where $w$ is the area ratio depending on $x_{v}$. The stiffness $K_{f}^{\prime}$ has a maximum value at $x_{v}=0$, while at large valve displacement

$$
\lim _{\left|x_{v}\right| \rightarrow \infty} K_{f}\left(x_{v}\right)=0
$$

The illustration of $K_{f}$ is shown in Figure 2 (nonlinear complex model). However, it is necessary to consider that the spool valve displacement is limited due to physical constraints $\left(x_{v, \max }= \pm 0.01 \mathrm{~m}\right)$. Therefore, at $x_{v, \max }$ the parameter $K_{f}\left(x_{v, \max }\right)$ is modified to a large value. It guarantees that the valve does not cause saturation. The modified piecewise function $K_{f}\left(x_{v}\right)$ is shown in Figure 2 (Broken line saturation approximation). Although the piecewise modeling results an appropriate formulation, for control-oriented modeling purposes a polynomial approximation is used. Thus, $K_{f}$ is approximated by a tenth-order polynomial of $x_{v}$ on the domain $\left[-x_{v, \max },+x_{v, \max }\right]$.

$$
K_{f}\left(x_{v}\right)=p_{10} x_{v}^{10}+p_{9} x_{v}^{9}+\ldots+p_{1} x_{v}+p_{0}
$$

where $p_{i}$ are the coefficients of the polynomial. Figure 2 also shows the polynomial approximation $K_{f}\left(x_{v}\right)$.

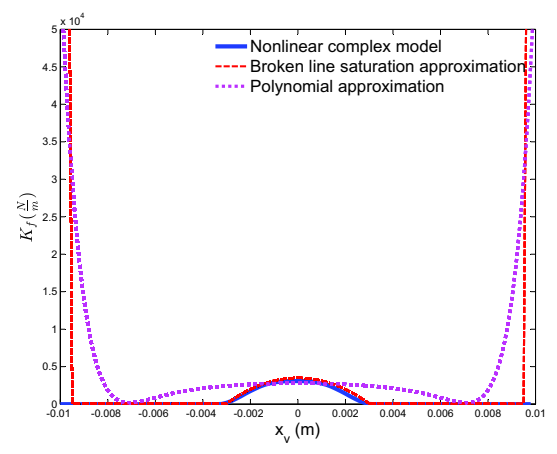

Fig. 2. Approximation of parameter $K_{f}$

Finally, the original dynamical equation (7) is transformed to the next form using (11)

$$
\ddot{x}_{v}=-2 D_{v} \omega \dot{x_{v}}-K_{f}\left(x_{v}\right) x_{v}+k_{v} \omega_{v}^{2} i
$$

\section{Control StRategy of the VAlve}

The valve control aims to track the reference spool displacement, defined by the controller of the hydromotor. This performance must be satisfied with the shortest settling time possible. Also the control input $i$ can only take three discrete values:

$$
i=\left\{-i_{\max }, i_{0}, i_{\max }\right\}
$$

where $i_{0}=0$. The control strategy is based on the Control Lyapunov Function. It is used to test whether a control input is able to stabilize the system.

Definition: Let a dynamical system be given the form

$$
\dot{x}(t)=f(x(t))+g(x(t)) u
$$


where $x(t) \in \mathbb{R}^{n}, u(t) \in \mathbb{R}$ and $f$ and $g$ are smooth vector fields and $f(0)=0$. A function $V$ is a Control Lyapunov Function if $V: \mathbb{R}^{n} \rightarrow \mathbb{R}$ is a smooth, radially unbounded and positive definite function.

The existence of such function implies that the system is asymptotically stabilizable at the origin, see [10].

The dynamical system has a differentiable ControlLyapunov Function if and only if there exists a regular stabilizing feedback $\mathrm{u}(\mathrm{x})$. It is called Artstein's theorem.

The tracking error of the control is given as follows:

$$
e=x_{v, r e f}-x_{v} .
$$

The derivative of this expression, assuming that the reference signal is constant for a given interval:

$$
\dot{e}=-\dot{x}
$$

Define the function $r$ and its derivative:

$$
\begin{aligned}
& r=\dot{e}+\alpha e=-\dot{x_{v}}+\alpha\left(x_{v, r e f}-x_{v}\right), \\
& \dot{r}=\ddot{e}+\alpha \dot{e}=-\ddot{x}_{v}-\alpha \dot{x}_{v},
\end{aligned}
$$

where $\alpha$ is a positive tuning parameter. Let the Lyapunov Function be given in the form

$$
V=\frac{1}{2} r^{2}
$$

This function is positive definite for every $r$. By deriving this function and substituting (17) the following equation is obtained:

$$
\dot{V}=r \dot{r}=\left(-\ddot{x_{v}}-\alpha \dot{x_{v}}\right)\left(-\dot{x_{v}}+\alpha\left(x_{v, r e f}-x_{v}\right)\right)
$$

Substituting the first row of (12) into (19):

$$
\left(2 D_{v} \dot{x_{v}}+K_{f} x_{v}-k_{v} i-\alpha \dot{x_{v}}\right)\left(-\dot{x_{v}}+\alpha\left(x_{v, r e f}-x\right)\right)=0
$$

By performing the multiplications, formally an equation of an ellipsoid for $\dot{x}_{v}$ and $x_{v}$ is obtained. The solution to the equation gives the limit of the controllable regions, wherein the states of the system can exist. The equation is written as follows:

$$
A_{e}{\dot{x_{v}}}^{2}+B_{e} x_{v}^{2}+C_{e} \dot{x_{v}} x_{v}+D_{e} \dot{x_{v}}+E_{e} x_{v}+F_{e}=0
$$

where $A_{e}, B_{e} \ldots F_{e}$ are the coefficients of the ellipsoid which are achieved by rearranging: $A_{e}=\alpha-2 D_{v}, B_{e}=$ $-K_{f}\left(x_{2}\right)-2 D_{v} \alpha+\alpha^{2}, C_{e}=-K_{f}\left(x_{2}\right) \alpha, D_{e}=k_{v} \omega_{v}^{2} i+$ $2 D_{v} \alpha x_{r e f}-\alpha^{2} x_{r e f}, E_{e}=K_{f}\left(x_{2}\right) \alpha x_{r e f}+k_{v} \omega_{v}^{2} i \alpha, F_{e}=$ $-k_{v} \omega_{v}^{2} i \alpha x_{r e f}$.

The parameter $\alpha$ must be tuned so that the system can reach the feasible states with the given control input. Note that $A_{e}, B_{e}, C_{e}, D_{e}, E_{e}, F_{e}$ are all functions of $\alpha$ so it has a significant effect on the shape of the set of the controllable regions. To achieve an acceptable performance, the aforementioned parameter must be selected carefully.

The states which can be stabilized by the control input are shown in Figure 3. Since the coefficients in (21) depend on the states, the ellipsoid is degenerated and opened on the $\dot{x_{v}}$, $x_{v}$ plane. The reference signal $x_{v, \text { ref }}$ can only take values

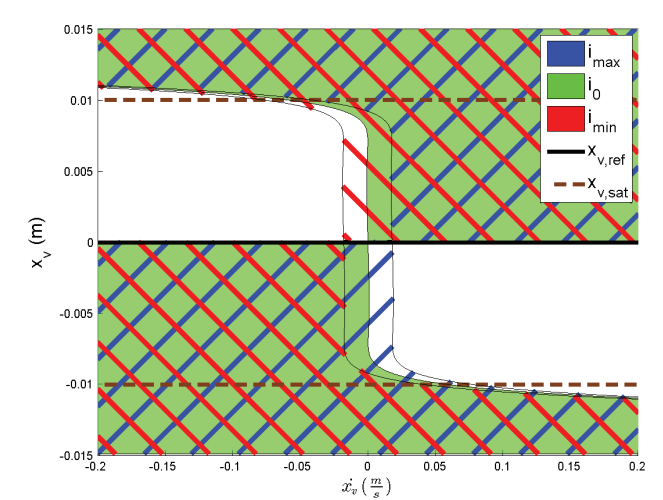

Fig. 3. Controllability regions of the discrete control inputs $\left(x_{v, \text { ref }}=0 \mathrm{~m}\right)$

between $\pm x_{v, s a t}$, which represent the saturation where the spool of the valve can not open more. The subsets where each control input can stabilize the plant are indicated with different colors. There are two domains where none of the control inputs can stabilize the system. However, this does not pose a problem since the system is stable, see (12). There are also domains where multiple inputs can take the system to the reference value. The control strategy exploits this feature to switch between control inputs.

The control algorithm for the spool valve is based on solving the Control Lyapunov Function. For every time step the control strategy calculates the values of the ellipsoids (21) by substituting the momentary values of the states and the reference signal for each discrete control input. The controller switches between input signals by choosing the appropriate solution. In the strategy the lowest value of the possible solutions is selected in order to guarantee reference tracking, i.e., $x_{v}$ tends toward $x_{v, r e f}$.

Assuming $E_{\max }, E_{0}, E_{\min }$ are the solutions of the ellipsoid equations (21) for $i_{\max }, i_{0}, i_{\min }$ respectively, the control algorithm can be formulated mathematically as follows:

$$
i=\left\{\begin{array}{ccc}
0 & \text { when } & \left\{E_{\text {max }}, E_{0}, E_{\text {min }}\right\} \geq 0 \\
i_{\text {max }} & \text { when } & \min \left\{E_{\text {max }}, E_{0}, E_{\text {min }}\right\}=E_{\text {max }} \\
i_{0} & \text { when } & \min \left\{E_{\text {max }}, E_{0}, E_{\text {min }}\right\}=E_{0} \\
i_{\text {min }} & \text { when } & \min \left\{E_{\text {max }}, E_{0}, E_{\text {min }}\right\}=E_{\text {min }}
\end{array}\right.
$$

For energy saving considerations, the control strategy presented above shall be augmented with an additional criterion. If the reference torque on the high level $M_{r e f}$ is a predefined small value, the control input is always set at zero. This criterion is necessary because otherwise the output $x_{v}$ would fluctuate around the reference $x_{v, r e f}$, which is zero at this point and the controlled system would never reach equilibrium.

\section{RoBUST CONTROL DESIGN OF THE HYDROMOTOR}

The actuator can be separated into two subsystems: the hydromotor (high level) and the valve (low level), which are interconnected. The goal of the hydromotor control is to track a reference torque $M_{r e f}$. The output signal of the highlevel controller $K_{a c t, u p}$ is a reference spool displacement 
$x_{v, r e f}$, which must be realized by the valve. The tracking of this reference signal is ensured by the low-level controller $K_{\text {act,low }}$, which computes discrete values of current $i$ on the solenoids, which cause the displacement of the spool.

In case of the independent control design the global stability of the controlled interconnected system must be ensured. A possible solution to guarantee the global stability of the individually stable systems is to prove the existence of a Common Lyapunov Function. In this paper the global stability of the system is guaranteed by the robust control design of the high-level control. In the design method the inaccuracy of the low-level tracking control is incorporated, which guarantees the interaction in the hierarchy. Moreover, other uncertainties of the actuator are considered in the robust control method.

In the following the robust control design of the upperlevel hydromotor is presented. The purpose of the control design is to guarantee the tracking of the reference torque $M_{r e f}$ by an appropriate valve motion $x_{v}$, which is physically realized by the low-level controlled valve system. Another important goal of the robust control design is to guarantee the global stability of the entire controlled actuator. First uncertainties of the actuator is detailed and second the robust $\mathcal{H}_{\infty} / \mu$ design is proposed.

\section{A. Uncertainties of the actuator}

1) Inaccuracy of low-level control: The aim of the analysis is to formulate the maximum tracking error of the lowlevel control. The result is incorporated in the design of the high-level robust control. Thus, the effect of the valve positioning inaccuracy is minimized.

The process of the analysis is the following. Several simulations are performed using different initial values $x_{v}(0)$, $\dot{x}_{v}(0)$ and reference position $x_{v, r e f}$. The intervals of the initial values are $x_{v}(0)=-0.01 m \ldots 0.01 m, \dot{x}_{v}(0)=$ $-0.1 \mathrm{~m} / \mathrm{s} \ldots 0.1 \mathrm{~m} / \mathrm{s}$ and $x_{v, r e f}=-0.01 \mathrm{~m} \ldots 0.01 \mathrm{~m}$. In each case the maximum tracking error is calculated. The

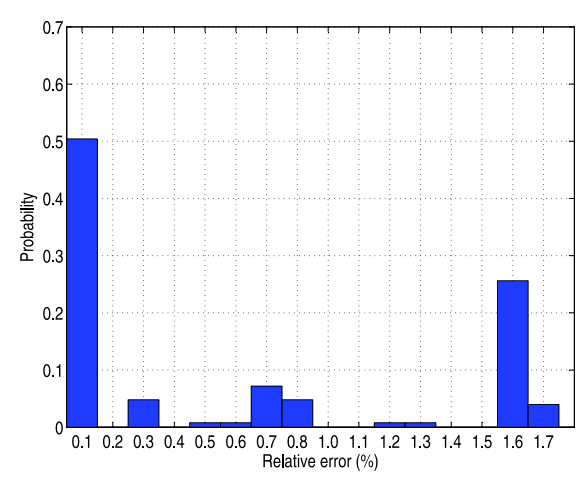

Fig. 4. Relative frequency of valve positioning

statistical results of the analysis are illustrated in Figure 4. It can be stated that the relative error of the valve positioning is reasonable. The error is below $0.1 \%$ and the maximum value is $1.7 \%$.

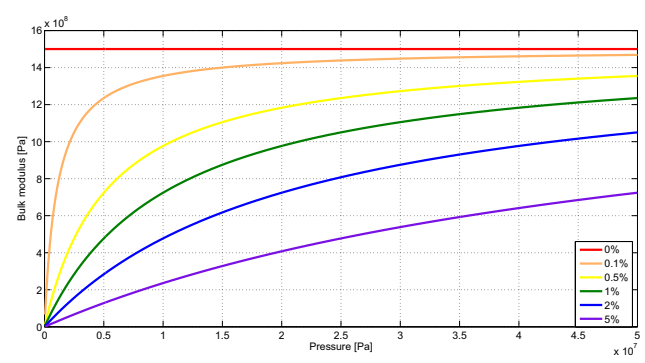

Fig. 5. Bulk modulus with different air contents

The results of the simulation-based statistical analysis are used for the modeling of an uncertainty in a multiplicative form. In the robust control design the worst case scenario is considered.

2) Uncertainty of the Bulk modulus: The Bulk modulus $\beta_{E}$ of the system (6) is an important physical parameter in the behavior of the hydromotor. It depends on several parameters, such as pressure and entrapped air. Generally, the pressure dependence at constant temperature can be formulated as $\beta_{E}=-V_{0} \frac{\partial p}{\partial V}$, where $V_{0}$ is the initial volume and $p$ is the pressure of the chamber.

Furthermore, $\beta_{E}$ depends significantly on the percentage of entrapped air in the system [9]. It seriously affects system performance in terms of loss of hydraulic power, slower response time, degradation in accuracy and the change in natural frequencies, which may cause stability issues [11]. When air is present in the system, the bulk modulus can be considered as two springs, connected in series:

$$
\frac{1}{\beta_{E}}=\frac{1}{\beta_{\text {fluid }}}+\frac{V_{\text {air }}}{V_{\text {total }}} \frac{1}{\beta_{\text {air }}}
$$

The adiabatic bulk modulus of air can be written as follows:

$$
\beta_{\text {air }}=\frac{c_{p}}{c_{v}} p=1.4 p
$$

where $c_{p}$ and $c_{v}$ are heat capacities at constant pressure and constant volume, respectively. Let $s=V_{\text {air }} / V_{\text {total }}$ be the percentage of air in the system. Using the expressions above, (23) can be written into the following form:

$$
\frac{1}{\beta_{E}}=\frac{1}{\beta_{\text {fluid }}}+\frac{s}{1.4 p}
$$

The connection between pressure and air content is illustrated in Figure 5.

It can be stated that $\beta_{E}$ is an important uncertain parameter of the system, which must be handled, see [12]. To formulate $\beta_{E}$ as a real parametric uncertainty, it is written in a lower linear fractional transformation (LFT) form:

$$
\beta_{E}=\bar{\beta}_{e}\left(1+d_{e} \delta_{e}\right)=\mathcal{F}_{l}\left(\left[\begin{array}{cc}
\bar{\beta}_{e} & 1 \\
d_{e} \bar{\beta}_{e} & 0
\end{array}\right], \delta_{e}\right)=\mathcal{F}_{l}\left(M_{e}, \delta_{e}\right)
$$

In the LFT structure the relationship between the output and the input of the block $M_{e}$ is $\tilde{y}_{e}=\bar{\beta}_{e} \tilde{u}_{e}+u_{e}$, while the uncertainty block $\delta_{e}$ is pulled out of the equation. $\bar{\beta}_{e}$ denotes the nominal value of the parameter and $d_{e}$ is a scalar, 
which represents the percentage of variation that is allowed for a given parameter around its nominal value. Moreover, $-1 \leq \delta_{e} \leq 1$ determines the actual parameter deviation. In the formulation of parametric uncertainties, $\delta_{e}, i \in(e)$ block must be pulled out of the motion equations.

The formulated $\tilde{y}_{e}$ output is used in (3) to express the parametric uncertainty of the system as follows:

$$
\begin{aligned}
\dot{p}_{L} & =\frac{4 \bar{\beta}_{e}}{V_{t}}\left(\bar{K}_{q} x_{v}-\bar{K}_{c} p_{L}-V_{p} \varphi+c_{l 1} \dot{\varphi}-c_{l 2} p_{L}\right)+ \\
& +\frac{4}{V_{t}}\left(\bar{\beta}_{e} u_{q}-\bar{\beta}_{e} u_{c}+u_{e}\right)
\end{aligned}
$$

\section{B. Robust $\mathcal{H}_{\infty} / \mu$ control design}

After the formulation of uncertainties, the robust control design of the hydromotor is presented. The purpose of the control is to guarantee the tracking performance of the system, formulated as follows:

$$
z=M_{\text {ref }}-M_{a c t} ; \quad|z| \rightarrow \min
$$

where $M_{r e f}$ is a reference torque signal, which is defined by the vehicle dynamic control. The goal of the controller is to guarantee criterion (28) against parameter uncertainties and disturbances (sensor noise and external load).

In the state-space representation, on which the control design is based, the parametric uncertainty and the inaccuracy of the low-level control are involved. Modifying the original system description (6) and considering the formulated performance (28), the hydromotor state-space representation is formed as:

$$
\begin{aligned}
\dot{x}_{H M} & =A_{H M, u} x_{H M}+B_{1 H M, u} w+B_{2 H M, u} u \\
z_{H M} & =C_{1} x_{H M}+D_{1,1} w \\
y_{H M} & =C_{H M} x_{H M}
\end{aligned}
$$

where the state vector, the disturbance and the control input are $x_{H M}=\left[\begin{array}{lll}p_{L} & \dot{\varphi} & \varphi\end{array}\right]^{T}, w_{u}=$ $\left[\begin{array}{llll}M_{\text {dist }} & M_{\text {ref }} & u_{e} & w_{n}\end{array}\right]^{T}$ and $u_{u}=x_{v}$, respectively.

In $\mathcal{H}_{\infty} / \mu$ control design several weighting functions are formulated which guarantee a balance between the performances and scale the different signals of the system. Figure 6 illustrates the closed-loop interconnection structure of control design.

The performance $z$ is considered with a weighting functions in the following form: $W_{z}=\left(\alpha_{1} s+\alpha_{0}\right) /\left(T_{1} s+T_{0}\right)$, where $\alpha_{1}, \alpha_{0}$ and $T_{1}, T_{0}$ are design parameters. The role of $W_{\text {dist }}$ and $W_{\text {ref }}$ is to scale torque disturbance signal $M_{\text {dist }}$ and reference torque $M_{r e f}$. The control system requires the measurement of tracking error $M_{r e f}-M_{a c t}$, as shown in Figure 6. The sensor noise $w_{n}$ of the measured signal is considered with weighting function $W_{n}$, which gives information about the bound of noise amplitude. Two uncertainty blocks are involved in the closed-loop interconnection structure. $\Delta_{r}$ incorporates the parametric uncertainty of the system, while $\Delta_{m}$ represents the uncertainty on the control input signal, which is derived from the imprecise realization of $x_{v}$ during low-level control. $W_{u}=\left(\alpha_{u, 2} s^{2}+\alpha_{u, 1} s+\right.$ $\left.\alpha_{u, 0}\right) /\left(T_{u, 1} s^{2}+T_{u, 1} s+T_{u, 0}\right)$ scales the bound of input

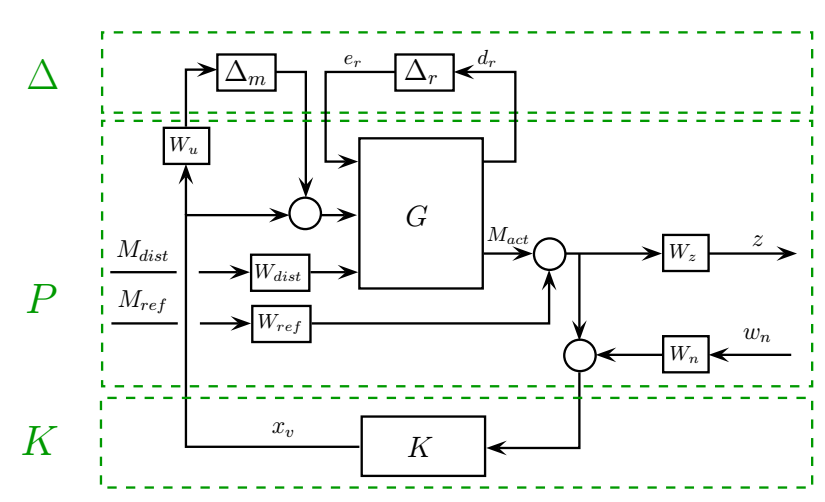

Fig. 6. Closed-loop interconnection structure

multiplicative uncertainty, where $\alpha_{u, 2}, \alpha_{u, 1}, \alpha_{u, 0}$ and $T_{u, 2}$, $T_{u, 1}, T_{u, 0}$ are design parameters.

In the robust $\mathcal{H}_{\infty} / \mu$ control design the controller synthesis problem is the following. Find a controller $K$ such that

$$
\mu_{\tilde{\Delta}}(M(i \omega)) \leq 1, \forall \omega \Leftrightarrow \min _{K \in \mathcal{K}_{\text {stab }}}\left[\max _{\omega} \mu(M(i \omega))\right]
$$

where $\mu$ is the function of the structured singular value of the system $M(i \omega)$ with a given uncertainty set $\tilde{\Delta}=$ $\operatorname{diag}\left[\Delta_{r}, \Delta_{m}, \Delta_{p}\right] . \Delta_{r}$ represents the parametric uncertainties, $\Delta_{m}$ describes the unmodelled dynamics and $\Delta_{p}$ is a fictitious uncertainty block, which incorporates the performance objectives into the $\mu$ framework.

The optimization problem can be solved in an iterative way by using scaling components. For fixed $K$ the problem of finding scaling components $D$ and $G$ is based on optimization problems. For calculated scaling components the problem of finding controller $K(s)$ leads to another optimization step. The procedure is called a standard $D, G-K$ iteration. The optimization problem is intractable in most cases, but an ad hoc algorithm has been developed, see [13].

\section{Demonstration example}

In this section the operation of the electro-hydraulic actuator is presented through a simulation example. The maximum spool valve displacement is $\left|x_{v, \text { sat }}\right|=0.01 \mathrm{~m}$, the discrete current inputs are $i=\{-0.35 ; 0 ; 0.35\} A$.

The reference torque signal $M_{r e f}$ is generated by the vehicle dynamic control. The torque tracking performance of the actuator is shown in Figure 7(a). In most of the simulation the difference between $M_{a c t}$ and $M_{r e f}$ is sufficiently low as illustrated in Figure 7(b). The relative tracking error is approximately $1 \%$. The tracking error only increases at high reference torque values and it is proportional to the magnitude of the reference signal. Noise on the torque measurement shown in Figure 7(c) does not have a significant effect on the tracking performances. Thus, the undesirable sensor noise can be rejected by the designed robust $\mathcal{H}_{\infty} / \mu$ control.

The valve positioning is shown in Figure 7(d). The lowerlevel operates with high precision, and does not exceed the saturation limit of the actuator. The control current of the valve system $i$ is found in Figure 7(e). The Figure 7 shows 


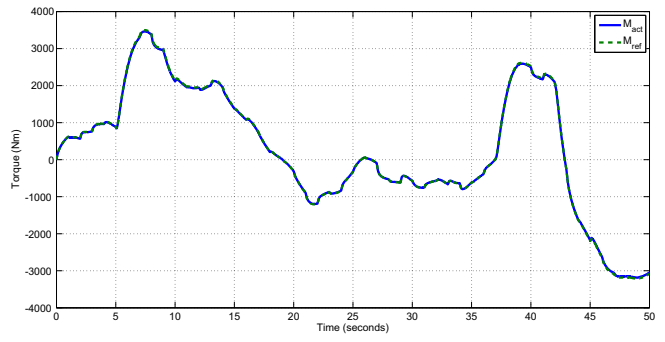

(a) Active torque of hydromotor

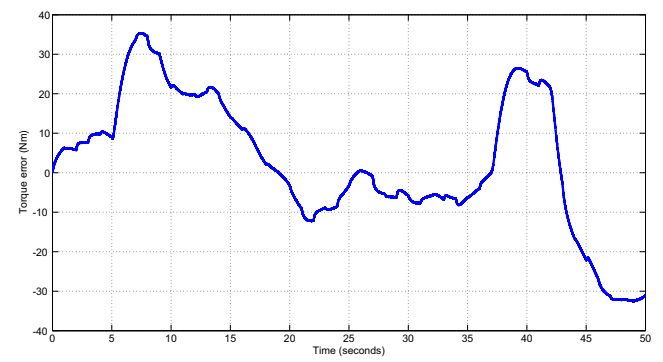

(b) Tracking error of hydromotor torque

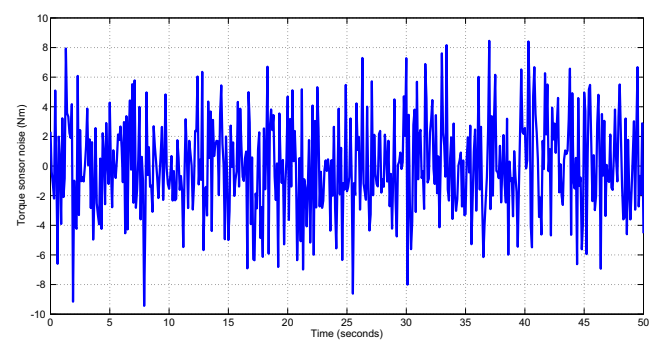

(c) Sensor noise on torque

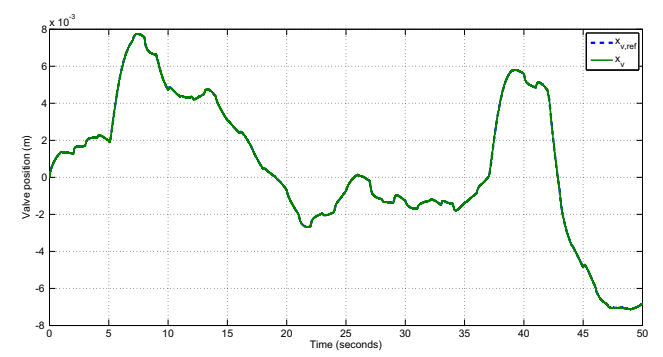

(d) Motion of electrohydraulic valve

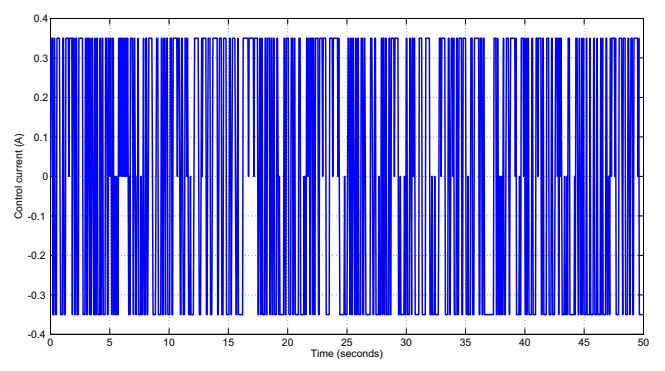

(e) Control input $i$

Fig. 7. Time responses of the closed-loop actuator that the low-level control is able to work adequately with fixed input values.

\section{CONCLUSION}

The paper has proposed the control design of an electrohydraulic actuator. The design is in line with the concept of hierarchical control systems. The control-oriented model of the hydromotor is formed as a linear system while the valve is a polynomial system. The valve model has a state constraint for the spool displacement due to physical considerations and it uses the Control Lyapunov Function to calculate discrete input current values. The hydromotor control is based on the $\mathcal{H}_{\infty} / \mu$ method, in which the inaccuracy of the lower-level control, parametric uncertainty and disturbances are incorporated. Thus, it guarantees the stability of the entire system. The advantage of this modular design is that the different requirements can be guaranteed for smaller-complexity subsystems. Simulation results prove that the control system can effectively track the reference torque in reasonable bounds, while the constraint of the system is not violated.

\section{ACKNOWLEDGEMENT}

The research has been conducted as part of the project TÁMOP-4.2.2.A-11/1/KONV-2012-0012: Basic research for the development of hybrid and electric vehicles. The Project is supported by the Hungarian Government and co-financed by the European Social Fund.

\section{REFERENCES}

[1] J. M. Hollerbach, I. W. Hunter, and J. Ballantyne, "A comparative analysis of actuator technologies for robotics," in The robotics review, vol. 2. MIT Press Cambridge, 1992, pp. 299-342.

[2] G. P. Liu and S. Daley, "Optimal-tuning nonlinear PID control of hydraulic systems," Control Engineering Practice, vol. 8, no. 9, pp. 1045-1053, 2000.

[3] M. G. Skarpetis, F. N. Koumboulis, and M. P. Tzamtzi, "Robust control techniques for hydraulic actuators," IEEE Mediterranean Conference on Control and Automation, vol. 15, 2007.

[4] P.-C. Chen and An-Chyauhuan, "Adaptive sliding control of active suspension systems with uncertain hydraulic actuator dynamics," Vehicle System Dynamics, vol. 44, no. 5, pp. 357-368, 2006.

[5] N. Niksefat and N. Sepehri, "Robust force controller design for an electro-hydraulic actuator based on nonlinear model," IEEE International Conference on Robotics and Automation, pp. 200-206, 1999.

[6] _ _ "Design and experimental evaluation of a robust force controller for an electro-hydraulic actuator via quantitative feedback theory," Control Engineering Practice, vol. 8, pp. 1335-1345, 2000.

[7] J. Jun, K. Kanaoka, and S. Kawamura, "Cascaded feedback control scheme for trajectory tracking of robot manipulator systems with actuator dynamics," Advanced Robotics, vol. 24, pp. 879-902, 2010.

[8] B. Sulc and J. A. Jan, "Non linear modelling and control of hydraulic actuators," Acta Polytechnica, vol. 42, no. 3, pp. 173-182, 2002.

[9] H. E. Meritt, Hydraulic control systems. John Wiley \& Sons Inc., 1967.

[10] E. D. Sontag, "A 'universal' construction of Artstein' on nonlinear stabilization," Systems and Control Letters, vol. 13, pp. 117-123, 1989.

[11] H. Y. J. Wang, G. Gong, "Control of bulk modulus of oil in hydraulic systems," IEEE/ASME International Conference on Advanced Intelligent Mechatronics, 2008.

[12] M. Karpenko and N.Shapehri, "Fault-tolerant control of a servohydraulic positioning system with crossport leakage," IEEE Transactions on Control Systems Technology, vol. 13, pp. 155-161, 2005.

[13] A. Packard and J. Doyle, "The complex structured singular value," Automatica, vol. 29, no. 1, pp. 71-109, 1993. 